Polymer Journal, Vol. 39, No. 6, pp. 589-592 (2007)

(C) 2007 The Society of Polymer Science, Japan

\title{
Alignment and Micropatterning of Carbon Nanotubes in Polymer Composites Using Modulated Magnetic Field
}

\author{
Guangzhe Piao, ${ }^{1}$ Fumiko Kimura, ${ }^{1}$ Tatsuhiro TaKahashi, ${ }^{2}$ Yoji Moritani, ${ }^{2}$ Hiroshi AwAno, ${ }^{2}$ \\ Shigeki NimORI, ${ }^{1}$ Kaoru TsudA, ${ }^{3}$ Koichiro YONETAKe, ${ }^{2}$ and Tsunehisa KImURA ${ }^{1,4, \dagger}$ \\ ${ }^{1}$ Tsukuba Magnet Laboratory, National Institute for Materials Science, 3-13 Sakura, Tsukuba 305-0003, Japan \\ ${ }^{2}$ Department of Polymer Science and Engineering, Yamagata University, Jonan, Yonezawa 992-8510, Japan \\ ${ }^{3}$ Nano Frontier Corporation, 3-10-6-105 Oosaki, Ota-ku, Tokyo 141-0032, Japan \\ ${ }^{4}$ Department of Applied Chemistry, Tokyo Metropolitan University, Minami-ohsawa, Hachioji 192-0397, Japan
}

(Received December 12, 2006; Accepted March 12, 2007; Published April 23, 2007)

\begin{abstract}
A facile method of simultaneous alignment and micropatterning of multi-wall carbon nanotubes (MWCNTs) is presented. The method uses the combination of the magnetic alignment and patterning techniques. Due to their diamagnetic nature, MWCNTs suspended in a liquid medium are trapped at the minima of the magnetic field created by a field modulator and at the same time they align due to their magnetic anisotropy. The achieved patterning and alignment is fixed by solidification of the matrix. The alignments both parallel and perpendicular to the film surface have been achieved. [doi:10.1295/polymj.PJ2006191]

KEY WORDS Magnetic Alignment / Magnetic Micropatterning / Carbon Nanotube / Magnetic Field Modulation /
\end{abstract}

Carbon nanotubes (CNTs) have been a focus of much research interest due to their remarkable properties. ${ }^{1-4}$ Nanocomposites made from CNTs and polymer offer the promise of composites with high electrical, thermal, optical, and mechanical properties. Since the CNTs possess high anisotropy, their alignment in the composites is the key to enhancement of these properties.

A number of studies have been reported on the alignment of CNTs including magnetic methods. ${ }^{5-7}$ The advantages of using magnetic fields are that it is possible to control the direction of CNTs with respect to the film surface. For example, it is possible to align CNTs parallel or perpendicular to the film surface: in fact, it is possible to align CNTs at any angle to the film surface.

In addition to the alignment, the patterning is also an important issue for the application to devices. ${ }^{8}$ So far, lithography and microcontact printing techniques have been used for micropatterning. In the previous papers, it has been reported that the magnetic method is also useful for patterning particles, ${ }^{9,10}$ as well as simultaneous alignment ${ }^{11}$ in the case that the particles are magnetically anisotropic.

In this paper, we present a facile method of fabricating polymer-based composites in which multi-wall carbon nanotubes (MWCNTs) form a line pattern with alignments. Polymers used are polyimide (PI), polystyrene (PS), and poly(vinyl alcohol) (PVA). Alignments parallel and perpendicular to the film surface are achieved.

\section{EXPERIMENTAL}

\section{Thermally Polymerized Film}

MWCNTs $\left(\mathrm{VGCF}^{\circledR}\right.$, generously donated by Showa Denko K.K.) were dispersed in $\mathrm{N}$-methylpyrrolidone (NMP), sonicated for $10 \mathrm{~min}$, then centrifuged at $3000 \mathrm{rpm}$ for $30 \mathrm{~min}$. The supernatant, containing dispersed MWCNTs, was then carefully decanted and mixed with an NMP solution of polyamic acid. The sample was placed in a modulated magnetic field, then heat-treated up to $70^{\circ} \mathrm{C}$ to evaporate the solvent while maintaining the orientation and patterning. It was then heated to $400{ }^{\circ} \mathrm{C}$ outside the magnet for the completion of imidization.

\section{Cast Film}

Preparation of cast film was performed under a magnetic field using two kinds of MWCNT suspensions as shown below.

Suspension 1: MWCNTs were dispersed into tetrahydrofuran (THF) with surfactant and placed in a YSNM-2000AR high pressure emulsifier (Yoshida Kikai Co., Ltd.), for fragmentation to 100 to $150 \mathrm{~nm}$ in diameter and 3 to $6 \mu \mathrm{m}$ in length. Polystyrene (PS) was added to the CNT suspension to obtain composite films with MWCNTs. An MWCNT suspension containing approximately $0.5 \%$ MWCNTs and 3.2\% PS in THF was used to prepare a sample of uniform alignment.

Suspension 2: $0.35 \%(\mathrm{~W} / \mathrm{V}) \mathrm{MWCNTs}\left(\mathrm{VGCF}^{\circledR}\right)$

${ }^{\dagger}$ To whom correspondence should be addressed (Tel: +81-42-677-2845, Fax: +81-42-677-2821, E-mail: kimura-tsunehisa@ c.metro-u.ac.jp). 


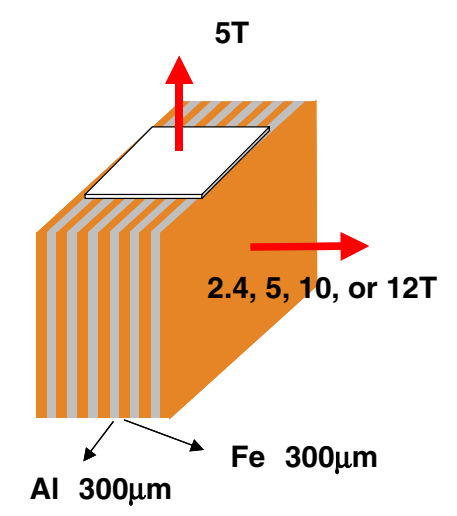

Figure 1. Optical microscopic photographs of uniform orientation of MWCNTs in PS composite film prepared under a 10-T horizontal magnetic field. The arrow indicates the direction of the applied magnetic field.

were dispersed in $1 \%$ sodium dodecyl sulfate (SDS) water solution by ultrasonication. 1 volume of supernatant of MWCNT suspension was mixed in 10 volumes of 5\% poly(vinyl alcohol) (PVA, MP Biomedicals, MW22,000) water solution at $50^{\circ} \mathrm{C}$.

\section{Magnetic Field}

The field modulators used were similar to those used in the previous study. ${ }^{9-11}$ They are composed of alternating aluminum and iron sheets 100 or $300 \mu \mathrm{m}$ thick, forming a layer structure of periodicity of 200 or $600 \mu \mathrm{m}$. The modulator was fixed in the center of two pole pieces of an electromagnet generating a horizontal field of $2.4 \mathrm{~T}$ or in the center of the superconducting magnets generating horizontal or vertical magnetic fields of 5,10 , or $12 \mathrm{~T}$. The sample was dropped on a cover glass placed on the modulator.

\section{Field Modulator}

The field modulators used in this study for the patterning are similar to those used previously and shown schematically in Figure 1. A field modulator is a block that is composed of alternating aluminum (100 or $300 \mu \mathrm{m})$ and iron $(100$ or $300 \mu \mathrm{m})$ layers. The magnetic field is applied horizontally or vertically. The horizontal magnetic field is applied perpendicular to the layers. In this case, the intensity of the magnetic flux density is weaker over the iron layers than the aluminum layers. On the other hand, if the magnetic field is applied vertically, the intensity is weaker over the aluminum layers. The modulation of the magnetic flux density persists approximately over a distance of about the pitch $(200$ or $600 \mu \mathrm{m})$ of the layer structure.

\section{RESULTS AND DISCUSSION}

\section{Uniform Alignment of MWCNTs}

A photograph of the MWCNT/PS composite film

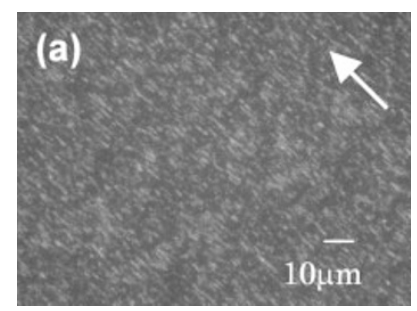

Figure 2. Schematic diagram of a field modulator. A block, composed of alternate aluminum $(100$ or $300 \mu \mathrm{m})$ and iron (100 or $300 \mu \mathrm{m}$ ) layers, was placed at the center of horizontal 2.4, 5, $10,12-\mathrm{T}$ or vertical 5-T magnet field.

prepared from a suspension under a uniform horizontal magnetic field is shown in Figure 2. MWCNTs are aligned parallel to the magnetic field. Between crossed polars, four extinctions appear during one revolution. The retardation of the composite film was estimated: The interference color of the film was blue under the $530 \mathrm{~nm}$ color plate. Since the blue color corresponds approximately to $780 \mathrm{~nm}$, the retardation of the film was roughly estimated as $250 \mathrm{~nm}$. The film thickness is approximately $30 \mu \mathrm{m}$ and the ratio of the MWCNTs in thickness is estimated at $10 \%$, resulting in birefringence of MWCNT $\Delta n=c a$. 0.1. This value is in agreement with the literature value. ${ }^{12}$ The birefringence of MWCNT is sufficiently large for use in optical devices, since the $\Delta n$ of calcite, widely used in optical devices, ${ }^{13}$ is 0.17 at $630 \mathrm{~nm}$.

\section{Patterning with Horizontal Alignment}

Figure $3 \mathrm{a}$ shows the patterning of MWCNTs in the polyimide composite films obtained with the modulated field $(200-\mu \mathrm{m}$ pitch) under the horizontal magnetic field $(2.4 \mathrm{~T})$. The black strips correspond to the MWCNTs. The boundary of the strips is not very clear because the thickness of the cover glass $(150 \mu \mathrm{m})$ used as a substrate is almost the same level as the estimated persistence length $(200 \mu \mathrm{m})$ and the applied field strength is not very high. Figures $3 b$ shows MWCNTs trapped in the PVA composite film obtained with the $600-\mu \mathrm{m}$-pitch modulator under the horizontal magnetic field of $10 \mathrm{~T}$. Trapped MWCNTs are aligned parallel to the applied magnetic field.

To confirm on which surface (aluminum or iron) the MWCNTs were trapped, we performed experiments using a modulator composed of 3 iron layers of $300-\mu \mathrm{m}$ wide and 2 aluminum layers of $300-\mu \mathrm{m}$ wide and sandwiched them alternately to form a $600-\mu \mathrm{m}$ pitch. The results are shown in Figure 4. Three lines of MWCNTs were observed, indicating that the MWCNTs were trapped on the iron surface. In this figure, the lines are denser than those displayed in Figure 3 because the suspension used here has a higher concentration of the MWCNTs. 


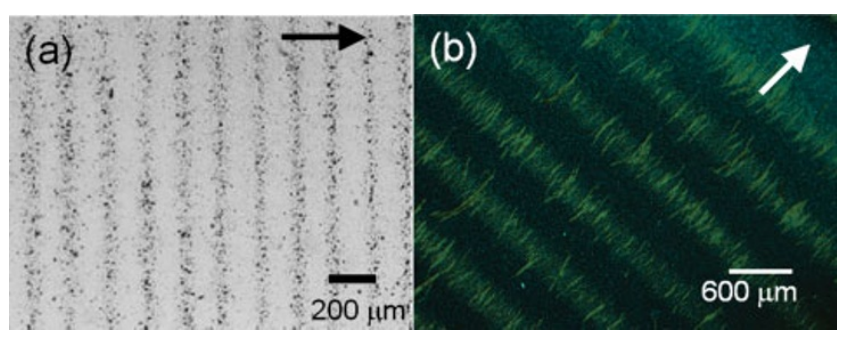

Figure 3. (a) PI composite film with patterned MWCNTs prepared by thermal polymerization and (b) PVA composite film with patterned MWCNTs prepared by casting, observed between crossed polars. The arrows indicate the direction of the applied magnetic field.

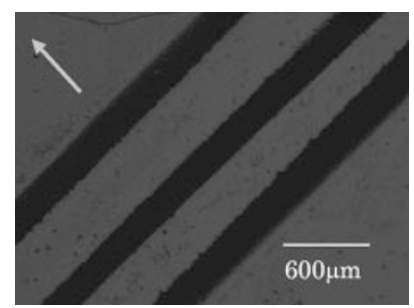

Figure 4. Three lines observed indicate that MWCNTs are trapped on the iron layers where the field strength is weaker. The arrow indicates the applied magnetic field.
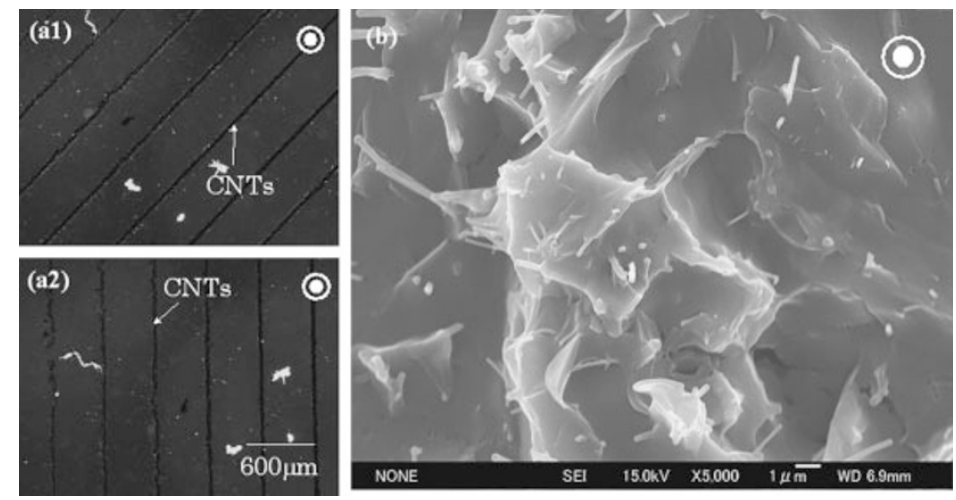

Figure 5. Trapping of MWCNTs perpendicular to the matrix PVA film prepared under a 5-T vertical magnetic field. Figures (a1) and (a2) are images taken between crossed polars indicated in the figure. MWCNTs are trapped on the aluminum layers. (b) SEM image of the alignment parallel to the applied magnetic field and perpendicular to the film surface. Spots indicate the direction of the magnetic field.

The concentration profile of magnetically isotropic particles under a modulated magnetic field has been discussed previously. ${ }^{14}$ In the present case, a modification is necessary because the particle under consid- eration has magnetic anisotropy. The concentration profile $c(x)$ of MWCNTs is described by the following equation:

$$
c(x)=c\left(x_{0}\right) \exp \left(\left(2 \mu_{0} k_{\mathrm{B}} T\right)^{-1} V\left(\chi_{\perp}+\chi_{\mathrm{a}} \cos ^{2} \theta-\chi_{\mathrm{m}}\right)\left(B^{2}(x)-B^{2}\left(x_{0}\right)\right)\right),
$$

where $\mu_{0}$ is the magnetic permeability of vacuum; $k_{\mathrm{B}}$, Boltzmann constant; $T$, the temperature; $V$, the volume of a MWCNT; $B(x)$, the magnetic flux density at location $x ; \chi_{\mathrm{a}}=\chi_{/ /}-\chi_{\perp}$ with $\chi_{/ /}$and $\chi_{\perp}$ being the magnetic susceptibilities of an MWCNT parallel and perpendicular to the long axis, respectively; $\chi_{\mathrm{m}}$, magnetic susceptibility of the liquid medium; $\theta$, the angle between the long axis and the magnetic field. If the MWCNT aligns parallel to the magnetic field $(\theta=0)$, the susceptibility part in the equation becomes $\chi_{/ /}-\chi_{\mathrm{m}}$, while if the MWCNT aligns perpendicular to the magnetic field $(\theta=\pi / 2)$, then it becomes $\chi_{\perp}-\chi_{\mathrm{m}}$. Because an MWCNT aligns parallel to the magnetic field before it reaches trapping zone, then $\chi_{/ /}-\chi_{\mathrm{m}}$ becomes relevant rather than $\chi_{\perp}-$ $\chi_{\mathrm{m}}$. The sign of $\chi_{/ /}-\chi_{\mathrm{m}}$ determines the location of the trap. If $\chi_{/ /}-\chi_{\mathrm{m}}<0$, that is, the $\chi_{/ /}$is relatively more diamagnetic than $\chi_{\mathrm{m}}$, then the MWCNTs are trapped at the place where the field strength is weaker. In Figure 4, we observed that MWCNTs are trapped on the iron layers where the field strength is weaker than the aluminum layers. This indicates that $\chi_{/ /}-$ $\chi_{\mathrm{m}}<0$.

\section{Patterning with Vertical Alignment}

Figure 5 shows the patterning of MWCNTs in the PVA composite film with a modulator $(600 \mu \mathrm{m}$ pitch $)$ under a $5 \mathrm{~T}$ vertical magnetic field. Figure 5a shows optical microscope images taken between crossed polars. MWCNTs formed narrow lines. They were trapped on the aluminum surface, since in the case of the vertical magnetic field, the flux density on the surface 
of aluminum is less dense than that on the surface of iron. When rotating the sample under the microscope, no extinctions were observed; as seen in Figure (a1) and (a2), the lines of MWCNTs remain dark under rotation by $45^{\circ}$. This indicates that the trapped MWCNTs were aligned parallel to the magnetic field and perpendicular to the film surface. Figure $5 \mathrm{~b}$ shows an SEM photograph showing the evidence of MWCNT alignment normal to the film surface: The heads of the MWCNTs protruded from the PVA film. The SEM observation is in agreement with the polarizing optical microscope observation. Because the open ends of MWCNT are expected to show a fast electron transfer rate similar to the graphite basal plane, this MWCNT composite could be applied to field emission devices.

\section{CONCLUSIONS}

We have reported a facile method of alignment and patterning of MWCNTs in a polymer composite. The method uses a field modulator placed in magnetic fields of 2.4 to $12 \mathrm{~T}$ that enables a simultaneous alignment/patterning of MWCNTs. The alignments that were both parallel and perpendicular to the film surface were achieved. The achieved alignment in a suspension was fixed by solidifying the suspending liquid through polymerization or evaporation of the solvent. This technique might be applied not only to MWCNTs, but also single-wall carbon nantubes (SWCNTs) as well as other nanotubes and nanorods that have magnetic anisotropy.
Acknowledgment. This work was partially supported by a Grant-in-Aid for Scientific Research on Priority Areas, "Innovative utilization of strong magnetic fields" (Area 767, No. 15085207) from MEXT of Japan.

\section{REFERENCES}

1. J. A. Misewich, R. Martel, Ph. Avouris, J. C. Tsang, S. Heinze, and J. Tersoff, Science, 300, 783 (2003).

2. N. de Jonge, Y. Lamy, K. Schoots, and T. H. Oosterkamp, Nature, 420, 393 (2002).

3. Y. Homma, Y. Kobayashi, and T. Ogino, Appl. Phys. Lett., 81, 2261 (2002).

4. E. Frackowiak and F. Beguin, Carbon, 39, 937 (2001).

5. M. Fujiwara, E. Oki, M. Hamada, Y. Tanimoto, I. Mukouda, and Y. Shimomura, J. Phys. Chem. A, 105, 4383 (2001).

6. T. Kimura, H. Ago, H. M. Tobita, S. Ohshima, M. Kyotani, and M. Yumura, Adv. Mater., 14, 1380 (2002).

7. M. J. Casavant, D. A. Walters, J. J. Schmidt, and R. E. Smalley, J. Appl. Phys., 93, 2153 (2003).

8. J. B. Hannon, A. Afzali, Ch. Klinke, and Ph. Avouris, Langmuir, 21, 8569 (2005).

9. T. Kimura, M. Yamato, and A. Nara, Langmuir, 20, 572 (2004).

10. T. Kimura, Y. Sato, F. Kimura, M. Iwasaka, and S. Ueno, Langmuir, 21, 830 (2005).

11. G. Piao, F. Kimura, and T. Kimura, Langmuir, 22, 4853 (2006).

12. D. Fry, B. Langhorst, H. Kim, E. Grulke, H. Wang, and E. K. Hobbie, Phys. Rev. Lett., 95, 038304 (2005).

13. E. O. Ammann and G. A. Massey, J. Opt. Soc. Am., 58, 1427 (1968).

14. T. Kimura, Polym. J., 35, 823 (2003). 